\title{
Study of American Option Pricing Based on Levy Process
}

\author{
Hong Zhang, Jie Zhu, Jian Guo, Li Zhou \\ School of Information, Beijing Wuzi University, Beijing, China
}

\section{Email address}

dr.yuwenjunxian@gmail.com (Hong Zhang)

\section{To cite this article:}

Hong Zhang, Jie Zhu, Jian Guo, Li Zhou. Study of American Option Pricing Based on Levy Process. American Journal of Applied Mathematics. Vol. 3, No. 3, 2015, pp. 151-156. doi: 10.11648/j.ajam.20150303.22

\begin{abstract}
After the 2008 financial crisis, the global derivatives trading volume in options proportion is growing, more and more investors build portfolios using options to hedge or arbitrage, our futures and stock options will soon open. Theoretical research of options is also changing, option pricing models under Levy processes developed rapidly. In this context, a review of the China's warrants market and the introduction of option pricing models can not only help us to reflect Chinese financial derivatives market regulation, but also to explore the option pricing theory for China's financial market environment. In the framework of Monte Carlo simulation pricing, we established mufti-Levy process option pricing models, the structural model for the given parameter estimation and risk-neutral adjustment method are discussed, the last part of this chapter is an empirical analysis of China warrants trading data in order to prove the validate of Levy models.
\end{abstract}

Keywords: Levy Stochastic Processes, Option Pricing Models, Chinese Warrants Market, American Option Pricing, Risk-Neutral Adjustment, Variance Reduction Techniques

\section{Introduction}

At present, both on OTC options or options trading, the path to the American option, Asian option as the representative of the dependent options are widely used. The Monte Carlo simulation technique used in this paper is difficult to directly price this kind of option. To solve this problem, this chapter introduces the American option pricing framework of the least squares method to improve the algorithm by using Levy process simulation of random path and give the improved algorithm. This paper checks it by using American warrant data and warrants validation data of China warrants market. In Section 1 of this chapter, by reviewing the previous American option, we discusses the difficulty of path dependent option pricing and the academic achievements in recent years Based on this, in Section 2, we introduce least square algorithm raise by Longstaff and Schwart (2001), this algorithm is the main framework of the Monte Carlo simulation technology to solve the American option pricing model. The pricing model in this paper is done in the framework of this framework. Based on the above results, in Section 3, a detailed description of the American option pricing method under the Levy process was made. According to the distribution characteristics of the random path of American option in the LSM method, the Levy-LARCH model is introduced by this method, instead of the normal distribution of the original path. In the 5.4 section, the Levy-LARCH-LSM model prices China's warrants depend on the path in China warrant market. Results show that, although the actual value of the warrant market in China has a large deviation compared with the no arbitrage assumption of the model, this method is better to some degree compared with the general LSM method, and the whole operation efficiency of the algorithm is ideal.

\section{The Pricing Problem of Path Dependent Options}

American options can be selected to execute on during the holding period, settled on the basis of the market price, so the pricing problem of the American option is a typical "optimal stopping problem". The main method to solve this problem are two binomial tree model and Monte Carlo simulation technique. Early option pricing model focuse on the two binomial tree method. Cox (1979) first proposed two binomial tree method with discrete time framework. This model eventually extended to the field of American option pricing (Zmeskal, 2010) through continuous improvement. But the use of two binomial tree model for pricing American options, we must do the new calculation respectively in every execution section, so when the random factor is excessive, the 
calculated pressure of two binomial tree method will be great. This problem is also called "dimension of crisis". In recent years, with the optimization of computer performance and Monte Carlo pricing algorithm, LSM, put forward by Longstaff and Schwart (2001), which is the representative of Monte Carlo simulation method, get more attention. Compared with the two binomial tree method, Monte Carlo simulation has better scalability, and is suitable for a plurality of random factor derivatives mode. This method gets more and more recognition, and this paper is under the framework of LSM, makes an empirical study on China American warrant pricing with Levy-G.ARCH price model.

Using Monte Carlo simulation method to solve the American option pricing starts at Carriere's article in 1996, but the article haven't solved the problem of expected cash flow estimation successfully. To solve this problem, Longstaff and Schwart (2001) using the least squares method, making an regression between the "future discounted cash flow " (discounted future cash flow) and the current "stock price", and reverse iterative recursive each of the expected cash flow, and get expected value according to the simulated path of update option cash flow, then to solve this problem. This method not only makes the pricing results more accurate, and the operation efficiency is very high, so it has good application value. Although Longstaff 'sand Schwart 's(2001) method solves the problem of dimension, but like any Monte Carlo pricing problem, through the use of better pricing algorithm, can increase simulation times in a short period of time, thereby reducing the degree of confidence, improve the results of the simulation results of variance. In recent years, study based on Monte Carlo simulation method is mainly embodied in two aspects: optimization of model simulation and optimization of the basic assets model. There are many research achievements of the optimization algorithm. Haugh and Kogan (2004), Bouchard (2004), Bouchard and Touzi (2004), Kim (2013) is extended to pricing algorithm. This article is mainly about the optimization of Least Squares Monte Carlo simulation method by using the Markoff chain, so as to improve the high dimension of American option pricing efficiency. Although research of the Least Squares Monte Carlo algorithm is relatively abundant, it is relatively lack of optimization on the basis of the asset model. As everyone knows, the volatility of the underlying asset characteristics plays a very important role in option derivatives, and Duan (1995) early introduce the GARCH model into European option pricing, and prove that the option pricing model under the time-varying volatility not only can better describe the volatility trajectory based assets, the results of its derivatives pricing model are significantly better than the traditional pricing model. Ghristoffersen gives the local equivalent martingale measure of risk neutral conversion by using a European option pricing model with Levy-GARCH. Because the American option pricing is more difficult, so the American option pricing model is late not perfect. Lars Stentoft (2012) introduced the GARCH model into American option pricing, and make a detailed description of the Monte Carlo simulation of American option pricing under the framework of GARCH method. Then he made a comparison between this model and a variety of mainstream American option pricing methods. The empirical results show that, both the index option or stock options, considering the change of the price of derivatives assets can be better described with of Least Squares Monte Carlo simulation method of GARCH effect, and improve the accuracy of American option pricing, but also found that the model there are many details need to be considered in this method, so there is still much room for improvement. In our country, the achievements about American options under the framework of the least squares method are limited. Wu Jianzu and Xuan Huiyu (2006), Sun Chunyan (2004), Yang Haijun and Lei Yang (2008) made empirical study of American option pricing by using the method of Monte Carlo. Zhang Lihua (2013) discusses the TS-LARCH model of American option pricing problem, but there is little research in option pricing with partial LARCH model and non normal model. Liu Qiang and Xiang Zan (2012) realized the research of least squares filtering option under LARCH model. He prove the validity of this method through the pricing of American option on the S \& P 100 index, All of the above research didn't taking the random number of non normal, non symmetrical jump characteristics and stock prices into account, while Marian and Seneta (1990), Kou (2002), Glosten (1993) has already introduced Levy process into the European pricing model, for fixing non normality yield sequence jump, and capturing the price and volatility, but so far, the study of American option jump model is still less. According to the above results and blank, we fix non normality rate series of based assets in GARCH model. By using a specific moment method to estimate the model parameters, and making the risk neutral pricing, we do the American option pricing analysis in the framework of simulation of least squares under Monte Carlo. we chose Levy-GARCH-LSM model ,GARGH-LSM model and the B-S model to make traditional the American warrant, Based on the research result of our American warrants, Bermuda option and S\&P 100 index of American option, we discuss ability to price of various model, and analyze whether China's warrants market obey the assumption of no arbitrage price.

\section{Empirical Analysis of the American Option Pricing in the Chinese Market}

From 2005 to 2009, in order to complete the reform of non tradable shares, the Shanghai and Shenzhen Stock Exchange traded a total of 55 warrants contracts, including 26 warrants and 29 equity warrants. The way of exercising right includes European, American and Bermuda. There are only 1 American airport and 14 Bermuda JTP1 warrants. Because the accuracy of option pricing model and pricing on the expiry date of the time interval distance has a significant relationship, we choose 160 days before the expiration of unified data. Because the SFC1 and JTP1 two warrants trading day is short, and the parameter estimation results are not stable and are not 
suitable for comparing and other rights certificate, the two warrants, makes an empirical analysis on the remaining 13 warrants. The following is the basic information of these warrants:

Table 1. Basic feature of the sample warrants.

\begin{tabular}{|c|c|c|c|c|c|c|}
\hline Abbreviation & The nature of warrants & Exercise way & Exercise price & Exercise proportion & Listing date & The starting date \\
\hline $\mathrm{ZXCl}$ & $\mathrm{C}$ & $\mathrm{B}$ & 78.13 & 0.5 & $200812 / 22$ & $2010 / 2 / 1$ \\
\hline $\mathrm{SFC} 2$ & $\mathrm{C}$ & $\mathrm{B}$ & 19 & 1 & $2007 / 6 / 29$ & $2008 / 5 / 19$ \\
\hline JTP1 & $\mathrm{P}$ & $\mathrm{B}$ & 15.1 & 1 & $2006 / 6 / 30$ & $2007 / 6 / 25$ \\
\hline EJC 1 & $\mathrm{C}$ & $\mathrm{B}$ & 5.5 & 1 & $2008 / 7 / 18$ & $2009 / 7 / 13$ \\
\hline $\mathrm{HQCl}$ & $\mathrm{C}$ & $\mathrm{B}$ & 7 & 1 & $2006 / 11 / 24$ & $2007 / 11 / 19$ \\
\hline JTP1 & $\mathrm{P}$ & A & 7 & 1 & $2005 / 12 / 23$ & $2006 / 3 / 23$ \\
\hline CWB 1 & $\mathrm{C}$ & $\mathrm{B}$ & 8.4 & 1 & $2008 / 3 / 7$ & $2009 / 3 / 2$ \\
\hline YGC 1 & $\mathrm{C}$ & $\mathrm{B}$ & 6.39 & 1 & $2006 / 4 / 3$ & $2008 / 3 / 27$ \\
\hline YGP 1 & $\mathrm{P}$ & $\mathrm{B}$ & 7.96 & 1 & $2006 / 4 / 3$ & $2008 / 3 / 27$ \\
\hline ZYP 1 & $\mathrm{P}$ & B & 10 & 1 & $2006 / 5 / 25$ & $2007 / 11 / 19$ \\
\hline GFC 1 & $\mathrm{C}$ & B & 3.95 & 1 & $2006 / 12 / 12$ & $2007 / 11 / 28$ \\
\hline HRP 1 & $\mathrm{P}$ & B & 3.73 & 1 & $2005 / 12 / 5$ & $2006 / 8 / 29$ \\
\hline CWB1 & $\mathrm{C}$ & B & 19.68 & 0.5 & $2008 / 3 / 4$ & $2010 / 2 / 25$ \\
\hline
\end{tabular}

1. Nature of warrants to $\mathrm{C}$ is bullish authority card, for $\mathrm{P}$ is put warrants.

2. The right way to B is the Bermuda authority card, for A is American warrants.

3. The strike prices of units for the yuan.

4. Line weight ratio of units for stock/copy

\subsection{The Normal Characteristics of the Test Data}

The reason of choosing Levy-G ARCH model mainly has two: one is the data of the volatility is time-varying, so we use LARCH model, and consider the characteristics of leverage volatility series. We select GJR-LARCH and EGARCH two kinds of LARCH models with partial; another reason is that the financial data does not follow a normal distribution, so the use of Levy process can better fit the real data. In order to directly confirm this two reasons, we first extract petrochemical CWB1 warrants the underlying stock volatility curve:
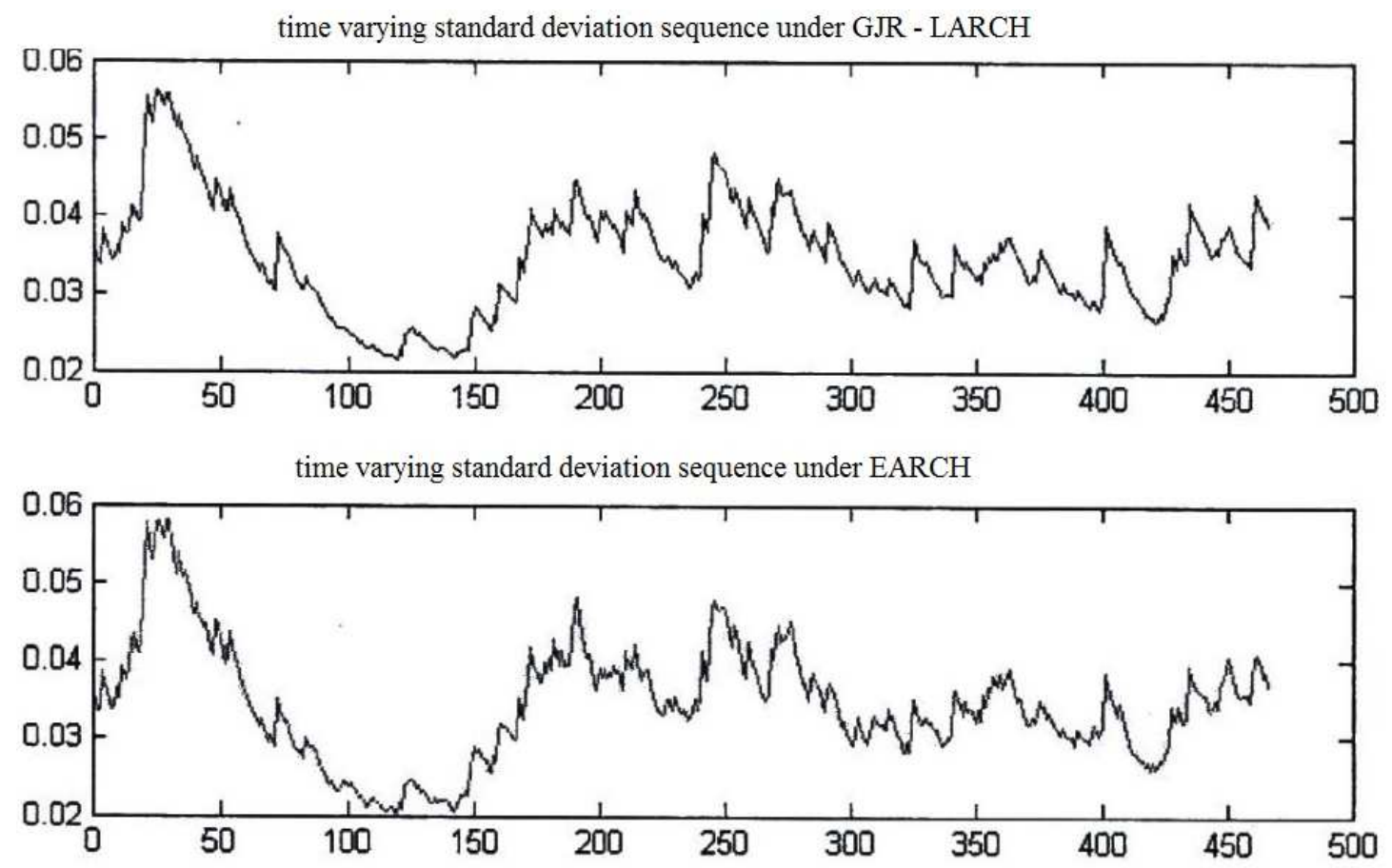

Fig. 1. Biased GARCH model volatility sequences.

As is pointed out in the volatility features of the time-varying standard deviation sequences by these two skewed GARCH models, we find that volatility has a strong characteristic of clump. Around the May, 2015, the volatility of petrochemical stocks is significantly higher than other periods, but in the latter two months, the volatility has fallen. This characteristic illustrates that GARCH model deals with the time-varying feature of the financial asset in an efficacious fashion. From the perspective of the data's skewness, the ascending rate of volatility is significant greater than the 
descending rate, which is mostly shown as the rapid rise and slow fall. This statistical feature is defined as the "leverage effect" of GARCH model. Based on the two features of the real-world data, we select the EGARCH and GJR-GARCH models that can describe the features above.
To demonstrate the non-normal property of the warrants' equity sequences, we present the moment estimators of the data to be estimated in Table 4. In the later part, we conduct the normality test.

Table 2. The statistical characteristics of the underlying securities.

\begin{tabular}{lllll}
\hline Data & Mean & Standard deviation & Skewness & Kurtosis \\
\hline Return rates & 0.00143251 & 0.00112683 & -0.107985262 & 4.592715772 \\
GJR-GARCH & -0.00094722 & 1.01086998 & 0.094674754 & 4.010116975 \\
EGARCH & -0.0040628 & 1.00669864 & 0.110112944 & 3.529748775 \\
SPY return rates & 0.00028722 & 0.00016460 & -0.0081 & 9.9437 \\
\hline
\end{tabular}

1. all the statistical characteristics of indicators for the 13 Stocks Index statistical arithmetic mean

2. where rate is the logarithm return rate of stock price, GJR-GARCH GJR-GARCH of the new information model, EGARCH EGARCH model information.

3. the last behavior of S\&P 100 index return (January 1, 1996 to August 30, 2013) of the statistical properties.

From the moment features of financial random statistics, the normal distribution is unbiased therefore the skewness is 0 . But for the real-world financial date, in terms of first order moment and second order moment, the skewness is not significantly equal to 0 significantly. Moreover, under the normal distribution, the kurtosis of random number is 4 . But the data of the logarithm return rate of the equity is greater than 4, i.e. in the real-world data, there exist a great deal of "Jump" events. So intuitively, the substitute of normal distribution by Levy process can help greatly simulate the financial random sequences, which is more approximate to the accurate pricing result.

Table 3. The statistical characteristics of the underlying securities.

\begin{tabular}{lllll}
\hline \multirow{2}{*}{ data } & Lillie test & \multicolumn{3}{l}{ JB test } \\
\cline { 2 - 5 } & $\begin{array}{l}\text { Rejection } \\
\text { rate }\end{array}$ & P values & $\begin{array}{l}\text { Rejection } \\
\text { rate }\end{array}$ & P values \\
\hline rate & $7 / 13$ & 0.148453671 & $8 / 13$ & 0.131676596 \\
GJR-GARCH & $4 / 13$ & 0.229133891 & $5 / 13$ & $0.1674 \mathrm{~b} 4051$ \\
EGARCH & $3 / 13$ & 0.296170307 & $4 / 13$ & 0.251944392 \\
\hline
\end{tabular}

1. Lilie is normal for the Lilliefors test. JB is Jarque-Bera normality test

2. the rejection rate indicates how many shares may decline to normal distribution the nullhypothesis, the significance level is $10 \%$. P value to two significant figures.

Liliefors is developed from the K-S test and can be used to conduct normality test. From the outcomes, we verity that no matter the Lilifors Test or the JB Test, more than half of the logarithm return rate sequences aren't subject to normal test. Since plenty of information can be expressed by the model's parameters after the filter of GARCH model, its information sequences are relatively characterized by normality. Even so, there are still random numbers which aren't subject to normal distribution within the framework of GARCH.

From the statistical characteristics, the skewed GARCH model can used to show the dynamic transformation characteristic of the volatility of financial data, while Levy process can also perform the non-normal feature of random number sequences. Thus, using the least square Monte Carlo simulation adjusted by Levy-GARCH model is more appropriate for simulating China's financial data.

\subsection{OLS Method Pricing Results Under the Levy - GARCH Model Correction}

Many studies show that, in China, only just a few Bermuda and American warrant holders choose to exercise the warrants before expiration date. As a result, we can regard these warrants as the European warrants to price. To study whether the European warrants, especially Black-Sholes-Merton model and other classical pricing models may underprice the warrant, we first use the Black-Sholes-Merton model to price the China's American and Bermuda warrants. Subsequently, we introduce the framework of the least square American option pricing to test the availability of the LSM framework in Chinese warrant market. To examine the non-normal characteristics of China's financial data and also to verify the significant effect of random distribution function on the Monte Carlo pricing method, we use two Levy processes---NIG and VF to price the warrants. Considering the time-varying feature of random number volatility, in the framework of LSM we use two types of GARCH models adjusted by Levy process - GJR-GARCH and EGARCH.

Study the pricing theory, and confirm the improvement of Levy-GARCH model to the pricing result.

Thus, we use the following 10 models to price the 13 American and Bermuda warrants.

By the comparison analysis of models, we address the following three questions:

1. Can Monte Carlo American option pricing formula precisely describe China's warrants? Is it applicable to use the European option pricing formula for the American and Bermuda option pricing in the context of few holders' exercising the warrants in advance in China?

2. Does the assumption that the return rate is subject to Levy process in pricing simulation help to significant improve the result in contrast to the assumption of normal distribution. If this improvement is significant, are there any differences between the varying Levy processes? 
3. Can the result of return rate volatility simulation be optimized by the GARCH model? Are there any differences between the varying GARCH models?

In answer to the question above, we use 7 pricing models and the simulation pricing of 38 warrants. From the outcomes shown, in general the differences of two Levy-LSM models are not obvious. The improvement seems better than the traditional Black-Scholes-Merton model. But Levy-GARCH-LSM pricing simulation has a much more precise result, with small variation between different GARCH models. But as a whole the ranks of different warrants by several models appears not always consistent. On the contrary in a few cases, the traditional Black-Scholes-Merton models are working.

To facilitate the models comparison and summarize the pricing result of warrants, we use the RMSER and AARE as the statistical indicators to evaluate the simulating pricing result and its errors deviated from the real prices. The construction of these indicators are:

$$
\begin{aligned}
& \text { RMSRE }=\sqrt{\sum_{i=1}^{N} \frac{\left(C_{i}^{\text {Model }}-C_{i}^{\text {Maket }}\right)^{2}}{N \times\left(C_{i}^{\text {Maket }}\right)^{2}}} \\
& \text { AARE }=\frac{\sum_{i=1}^{N}\left|C_{i}^{\text {Model }}-C_{i}^{\text {Maket }}\right|}{C_{i}^{\text {Maket }} \cdot N}
\end{aligned}
$$

Table 4. Comparison of pricing error between models.

\begin{tabular}{lcc}
\hline model & RMSRE & AARE \\
\hline Chinese American, Bermudan & Warrants Pricing Error & \\
B-S & 0.654027 & 0.539912 \\
LSM & 0.506878 & $0: 428039$ \\
NIG-LSM & 0.487575 & 0.405472 \\
VG-LSM & $0.4900 \$ 7$ & 0.407053 \\
NIG-GJR-LSM & 0.465277 & 0.380198 \\
VG-GJR-LSM & 0.464451 & 0.375035 \\
NIG-EGARCH-LSM & 0.467823 & 0.389590 \\
VG-EGARCH-LSM & 0.473374 & 0.387143 \\
S\&P100 index of American option pricing errors $(0.99<\mathrm{K} / \mathrm{S}<1.01)$ \\
Conditional B-S & 0.2013 & 0.2856 \\
Conditional B-S LSM & 0.2003 & 0.2841 \\
Conditional VG & 0.1997 & 0.2826 \\
Conditional VG-LSM & 0.1995 & 0.2823 \\
VG N-LARCH & 0.1345 & 0.2013 \\
VG-N-LARCH-LSM & 0.1312 & 0.1966 \\
CTS-N-LARCH & 0.1323 & 0.1988 \\
CTS-N-LARCH-LSM & 0.1305 & 0.1961 \\
S\&P 100 index American option pricing error $(0.95<\mathrm{K} / \mathrm{S}<0.99)$ Call in the \\
money & & \\
Conditional B-S & 0.2089 & 0.2962 \\
Conditional B-S LSM & 0.2087 & 0.2964 \\
Conditional VG & 0.2099 & 0.2954 \\
Conditional VG-LSM & 0.2078 & 0.2926 \\
VG-N-GARCH & 0.1086 & 0.1661 \\
VG-N-GARCH-LSM & 0.1084 & 0.1643 \\
CTS-N-GARCH & 0.1076 & 0.1612 \\
CTS-N-GARCH-LSM & 0.1076 & 0.1607 \\
\hline
\end{tabular}

From the pricing result, Black - Scholes - Merton model performance was significantly lower than other several models. So the first thing we can be sure is that even if the Bermuda warrants and American style warrants' card holder's trading behavior is similar to the European warrants, which almost do not exercise in advance, but the American options pricing formula should also consider the expected cash flow effects of exercise way, which cannot be used directly to European option pricing model. Monte Carlo simulation is also verified the American option pricing formula has a lot to improve on pricing accuracy, especially using the Levy random distribution NIG - LSM and VG - LSM model, the non-normal correction results compared to the ordinary has markedly improved the LSM model, but NIG and VG have similar effects to the promotion of the results. At last, introducing partial GARCH model to the end of the four groups of model reflects the best pricing accuracy. Among them, the VG - GJR - the LSM model has the best pricing accuracy. The results further illustrate the time-varying volatility on the importance of the option derivatives pricing.

Although the LSM model, Levy random process, partial GARCH model can promote our country's American style Warrants and the Bermuda warrants' pricing accuracy on different levels, but pricing accuracy of these models are still not ideal. RMSRE data is close to $50 \%$, but the results compared to the American options less than 20\% RMSRE indicators in major U.S. trading market still have a very big gap. With respect to China's warrants market pricing efficiency, Xiong and Yu (2011) and Hailin Zhou (2012) think in our country, market speculation is too active and trading system is not perfect by analyzing the price data and transaction data, These factors significantly deviated the warrants market in China from the efficient markets hypothesis, so the theory model set up under the hypothesis of no-arbitrage is difficult to estimate market price of warrants in China.

\section{Summary}

In the framework of Least Squares Monte Carlo method of American option pricing, we consider that financial sequence is partial and jumping, so we us the Levy process to fix normal distribution and introduce partial GARCH model to describe the financial volatility series gathering effect and leverage effect. Levy-GARCH-LSM model is established. According to the data of China's warrants market, we selected 8 pricing model to price the 12 warrants and 1 American warrant.the conclusions are as follows:

Although the majority of Bermuda warrants and American warrant holders in China will not exercise in advance, this kind of path dependent warrants is still not pricing by European option pricing model. LSM pricing model can estimate the expected return during each period, which gives reliable pricing results.

2. Because the yield sequence has statistical characteristics of "biased" , "thick tail" and " non normal", so under the framework of LSM model, using the" Levy" process to modify the financial random sequence, can effectively 
improve the estimation accuracy. NIG and the VG model can give very good fitting effect.

3. Considering the volatility of financial asset rate time-varying characteristics, using the GARCH model, especially offset GARCH model such as GJR-GARCH and EGARCH model can better describe the warrant duration of underlying asset volatility, which gives better pricing accuracy.

4. Levy-GARCH-LSM pricing model in China's warrants market was significantly better than the classical model in the past, but because of the lack of China's warrants market pricing efficiency, the model based on no arbitrage assumptions still are not sufficiently accurate pricing results.

Generally speaking, although Levy-GARCH-LSM pricing accuracy is still limited for the warrants market data in China, compared to the traditional LSM model, the modified model can still better capture the market volatility, and better simulate the jump characteristics of the market, and the pricing deviation is mainly due to China's warrants market microstructure issues. And through the empirical study, proved that this method is practical, efficient and stable for American option pricing.

\section{Acknowledgements}

This paper is funded by the project of National Natural Science Fund, Logistics distribution of artificial order picking random process model analysis and research (Project number: 71371033); and funded by intelligent logistics system Beijing Key Laboratory (No. BZ0211); and funded by scientific-research bases--- Science \& Technology Innovation Platform---Modern logistics information and control technology research (Project number: PXM2015_014214_000001); and funded by 2014-2015 school year, Beijing Wuzi University, College students' scientific research and entrepreneurial action plan project (No.68); and funded by Beijing Wuzi University, Yunhe scholars program (00610303/007); and funded by Beijing Wuzi University, Management science and engineering Professional group of construction projects. (No. PXM2015_014214_000039). University Cultivation Fund Project of 2014-Research on Congestion Model and algorithm of picking system in distribution center (0541502703).

\section{References}

[1] Koponen, I. Analytic approach to the problem of convergence of truncated Levy flights towards the Gaussian stochastic process [J]. Physical Review E, 1995, 52: 1197-1199.

[2] Lays Stentoft. American option pricing using simulation: an introduction with to the GARCH option pricing model[C]. CREATES working paper, 2012.

[3] Lehar A, Scheicher M, Schittenkopf C. GARCH vs. stochastic volatility: option pricing and risk management [J]. Journal of Banking \& Finance, 2002, 160(1): 246-256.

[4] Longstaff F A, Schwartz E S. Valuing American options by simulation: a simple least-squares approach [J]. The Review of Financial Studies, 2001, 14(1): 113-147.

[5] Lydia W. American Monte Carlo option pricing under pure jump Levy models [D]. Stellenbosch University, 2013.

[6] Kim J, Jang B G, Kim K T. A simple iterative method for the valuation of American options [J). Quantitative Finance, 2013, 13(6): 885-895.

[7] Chorro C, Guegan D, hyperbolic Lelpo F. Option pricing for GARCH-type models with innovation [J]. Finance, 2012, 12(7): 1079-1094.

[8] Christoffersen P, Jacobs K, Ornthanalai C. GARCH option valuation: and evidence [Z]. Aarhus University, Working Paper, 2012. theory

[9] Byun SJ, Min B. Conditional volatility and the GARCH option pricing model with non-normal innovations [J]. 3ournal of Futures Market, 2413, 33(1): 1-28.

[10] Carr P, Madan D B. Option valuation using the fast Fourier transform [J]. Journal of Computational Finance, 1999, 2(4): $61-73$.

[11] Carr P, Geman H, Madan D H and Yor M. The fine structure of asset returns: an empirical investigation [J]. Journal of Business, 2002, 75(2): 305-332.

[12] Carr P and Wu L R. The finite moment log stable process and option pricing [J]. Journal of Finance, 2003, 58(2): 753-777.

[13] Carriere J F. Valuation of the early exercise price for options using simulations and nonparametric regression[J]. Insurance: Mathematics and Economics, 1996, 19(1): 19-30. 